\title{
The Efficacy of Miniaturized Repetitive Transcranial Magnetic Stimulation in Patients with Depression
}

\author{
Sangmin Lee ${ }^{1,2}$, Kuk-In Jang ${ }^{1,2}$, Sejin Yoon ${ }^{3}$, Jeong-Ho Chae ${ }^{1,2,4}$ \\ ${ }^{1}$ Department of Biomedicine and Health Sciences, College of Medicine, The Catholic University of Korea, ${ }^{2}$ Institute of Biomedical Industry, \\ The Catholic University of Korea, Seoul, ${ }^{3}$ REMED Co., Ltd., Daejeon, ${ }^{4}$ Department of Psychiatry, Seoul St. Mary's Hospital, College of Medicine, \\ The Catholic University of Korea, Seoul, Korea
}

\begin{abstract}
Objective: The present study aimed to evaluate the efficacy of repetitive transcranial magnetic stimulation (rTMS) at a high frequency using a miniaturized device compared to standard rTMS and sham rTMS for the treatment of depression.

Methods: Fifty-four patients with depression were randomly assigned to either 15 days of miniaturized, standard, or sham rTMS. The stimulation consisted of 60 trains of 5 seconds at $10 \mathrm{~Hz}$ for 30 minutes. Clinical measures were assessed at baseline and on the final day of the stimulation.

Results: A repeated-measures analysis of variance revealed a significant main effect of time and a time by group interaction on Hamilton Rating Scale for Depression scores. There were no significant correlations between individual motor thresholds and changes of clinical outcomes. Our results revealed a significant reduction in the Hamilton Rating Scale for Depression in the miniaturized and standard groups compared to the sham group.

Conclusion: The antidepressant utility of miniaturized rTMS using subthreshold stimulation was comparable to that of standard stimulation.
\end{abstract}

KEY WORDS: Depression; Transcranial magnetic stimulation; Antidepressants.

\section{INTRODUCTION}

Promoting both existing treatment options and greater access to treatment may be more clinically significant than the development of entirely new treatment modalities, because many individuals who face depression receive no treatment for their symptoms. ${ }^{1)}$ Many alternative treatments for patients with major depressive disorder (MDD) have emerged in recent years. ${ }^{2)}$ Among these, repetitive transcranial magnetic stimulation (rTMS), a promising neuromodulatory technique, has been demonstrated to be safe and effective for the treatment for MDD. Previous studies have demonstrated the antidepressant ef-

Received: August 20, 2018 / Revised: October 29, 2018

Accepted: November 30, 2018

Address for correspondence: Jeong-Ho Chae

Department of Psychiatry, Seoul St. Mary's Hospital, College of

Medicine, The Catholic University of Korea, 222 Banpo-daero,

Seocho-gu, Seoul 06591, Korea

E-mail: alberto@catholic.ac.kr

ORCID: https://orcid.org/0000-0002-6070-9324 ficacy of high-frequency rTMS when applied to the left dorsolateral prefrontal cortex (DLPFC), specifically. ${ }^{3-7)}$

Many outpatients being treated for depression fail to complete their recommended course of treatment. ${ }^{8)}$ Because this premature termination can result in poorer outcomes (e.g., increased major depressive episodes), improving the continuity of treatment for these patients is likely critical to their care. ${ }^{9)}$ Standard rTMS protocols require daily stimulation for several weeks and may consequently lead to elevated treatment discontinuity rates. ${ }^{10)}$ Drop-out rates with conventional high frequency rTMS and sham rTMS are comparable, ${ }^{11)}$ as daily clinic visits are required for both and may be an obstacle to adequate treatment continuity. The use of miniaturized devices, which are smaller and lighter than standard ones, may thus serve as a good solution for outpatient treatment. Patients with MDD could potentially use these devices at home without regular visits to clinic, lowering rates of disuse and thus improving treatment adherence.

Treatment with rTMS is characterized by many varia-

(ㄷ) This is an Open-Access article distributed under the terms of the Creative Commons Attribution Non-Commercial License (http://creativecommons.org/licenses/by-nc/4.0) which permits unrestricted non-commercial use, distribution, and reproduction in any medium, provided the original work is properly cited. 
bles including stimulation intensity, frequency, the number of trains and their intervals, and the site of application. The importance of these variables to the effects of rTMS on depression remain unclear. Previous studies have suggested that suprathreshold rTMS stimulation is superior to subthreshold rTMS stimulation for the treatment of depression. ${ }^{12,13)}$ While subthreshold rTMS can suppress corticospinal excitability, its latent effects tend to be weaker than suprathreshold rTMS. ${ }^{14)}$ However, some studies have also indicated that subthreshold high frequency rTMS induces more lasting facilitation. ${ }^{15,16)}$ Further consideration of the intensity limits of miniaturized rTMS and the efficacy of subthreshold stimulation, as well as its association with individual motor threshold (MT), remains necessary before this treatment modality can be clinically validated.

Given this paucity of data on rTMS in patients with MDD, we report here results of the first trial of an experimental device designed to administer miniaturized rTMS to patients with MDD. This device, which consists of a smaller stimulator than standard one and a wearable rTMS coil, is used with the same protocol as a standard rTMS device. This study was designed to investigate the efficacy and safety of miniaturized rTMS treatment compared to standard and sham rTMS treatment. We further explored whether the efficacy of miniaturized rTMS was associated with individual MT.

\section{METHODS}

\section{Participants and Procedure}

Fifty-four patients who met the criteria for unipolar MDD, as outlined in the 5th edition of the Diagnostic and Statistical Manual of Mental Disorders (DSM-5) participated in this study between February 2015 and November 2016 at the Department of Psychiatry, Seoul St. Mary's Hospital, The Catholic University of Korea, Republic of Korea. Trained psychiatrists who were not directly associated with the present study conducted Mini-International Neuropsychiatric Interviews with all participants. ${ }^{17)}$ All participants were between 18 and 65 years of age, and had no active medical conditions. They were permitted to continue taking psychotropic medications and the doses of these medications were not changed during the rTMS treatment period. Participants with other current and/or lifetime Axis I psychiatric disorders, a history of epilepsy or brain surgery, substance use disorders, current preg- nancy or previous experience with rTMS were excluded. Participants with contraindications for magnetic stimulation (e.g., cardiac pace makers, implanted medication pumps, or hearing aids containing metal materials) were also excluded. For safety purposes, all participants underwent a brief electroencephalography (EEG) session to screen for epileptiform EEG abnormalities prior to rTMS treatment.

Prior to treatment, the participants were randomized to receive either standard rTMS, miniaturized rTMS, or sham rTMS. Participants underwent a clinical assessment on the first day of the study (pre-rTMS) and then after 15 sessions of rTMS (post-rTMS). Seven participants were dropped from the study due to non-compliance. The final sample consisted of 16 participants in the standard rTMS group, 17 participants in the miniaturized rTMS group, and 14 participants in the sham rTMS group (total participants, 11 men and 36 women; mean age, $34.81 \pm 11.41$ years; age range, 19-62 years). The Institutional Review Board of Seoul St. Mary's Hospital, College of Medicine, The Catholic University of Korea approved the study protocol (approval number KC14DDSE0479). All participants provided written informed consent prior to participation.

\section{Clinical Measures}

To determine the clinical efficacy of each rTMS treatment, trained raters, who were blinded to participants' clinical/treatment information, scored participants using the Clinical Global Impressions scale for Severity (CGI-S) and Improvement (CGI-I), the 17-item version of the Hamilton Rating Scale for Depression (HAM-D), ${ }^{18)}$ and the 14-item version of the Hamilton Rating Scale for Anxiety (HAM-A). ${ }^{19)}$ Depressive symptoms were also evaluated using the Beck Depression Inventory (BDI), ${ }^{20)}$ a well-validated, self-report assessment composed of 21 items. Response was defined as $\geq 50 \%$ decrease in HAM-D, and remission was defined as final HAM-D score $7 .^{21)}$

Secondary outcomes included the Sheehan Disability Scale (SDS) ${ }^{22)}$ and the visual analogue scale (VAS ${ }^{23)}$ to assess participants' functional impairment and pain, respectively.

\section{rTMS}

Standard rTMS was administered using TAMAS (REMED, Daejeon, Korea) with a figure-of-eight shaped coil (field strength $\sim 3$ Tesla). Prior to each rTMS session, participant 
MT was determined by detecting the lowest level of stimulation energy required to stimulate the motor cortex and produce five consecutive twitches of the right abductor pollicis brevis (APB) muscle. Stimulation was then applied at $110 \%$ of the individual MT. Stimulation was applied over the DLPFC, the location of which was determined in each participant by moving the TMS coil $5 \mathrm{~cm}$ anterior to the optimal surface site for activation of the right APB muscle. ${ }^{10,24)}$ The frequency of stimulation was set to $10 \mathrm{~Hz}$ for 5 seconds, with an inter-train interval of 25 seconds. Treatment sessions lasted for 30 minutes (60 trains) and included 3,000 pulses.

The miniaturized rTMS device, Brain Stim (REMED), contained a figure-of-eight, 80-mm diameter wearable rTMS coil connected to a downsized stimulator (dimensions, $430 \times 270 \times 89 \mathrm{~mm}$; weight, $4.5 \mathrm{~kg}$; Fig. 1). The maximum magnetic field generated by the stimulator was 2.5 Tesla. The magnetic stimulus had a biphasic waveform with a pulse width of approximately $320 \mathrm{~ms}$. As with standard rTMS administration, individual MT was determined by stimulating the motor cortex using TAMAS, and stimulation was applied at $110 \%$ of the individual MT. Unlike standard rTMS, which performs cooling by circulating the cooling oil inside the transducer, the miniaturized rTMS unit employed an air-cooling system. Although this system allowed for the device to be smaller, it insufficiently reduced coil heating. The safeguard against overheating was built into the system. The device stops automatically when the temperature of the coil exceeds $40^{\circ} \mathrm{C}$. The coil was replaced every 10 minutes during stimulation, and stimulation intensity was limited to $70 \%$ of maximum stimulator output for safety purposes. The location, frequency, and duration of stimulation with the mini- aturized rTMS were the same as with standard rTMS.

Sham stimulation was performed using the miniaturized rTMS device with a sham coil, which elicited no tactile sensation at the site of stimulation and induced no cortical stimulation. The device did, however, provide matched acoustic sensation. Each participant, regardless of assigned rTMS group, underwent 15 rTMS sessions on 15 consecutive weekdays in the outpatient rTMS room. Because this study was single-blind trial, rTMS operators were not blinded.

\section{Statistical Analyses}

Statistical analyses were performed using SPSS software (version 20; IBM Corp., Armonk, NY, USA). The level of significance was set at $p<0.05$. For all clinical measures under consideration, a Kolmogorov-Smirnov test of normality was performed. At baseline, group differences in clinical measures between the three rTMS groups were tested via an analysis of variance (ANOVA) and KruskalWallis tests. Group differences in sex and response were tested by chi-square tests. A repeated-measures ANOVA was used to analyze the effects of rTMS on HAM-D scores. Differences in HAM-D, HAM-A, and BDI changes between three rTMS groups were analyzed using ANOVA and post-hoc Scheffé test. Effect sizes are expressed as a partial eta-square $\left(\eta_{p}^{2}\right)$.

Group differences in individual MT between the standard and miniaturized rTMS groups were computed using independent $t$ tests. Pearson's correlations were also calculated to determine the association between the stimulation percentage of individual MT and changes to clinical outcomes in the standard and miniaturized rTMS groups. Changes in SDS and VAS were also analyzed us-
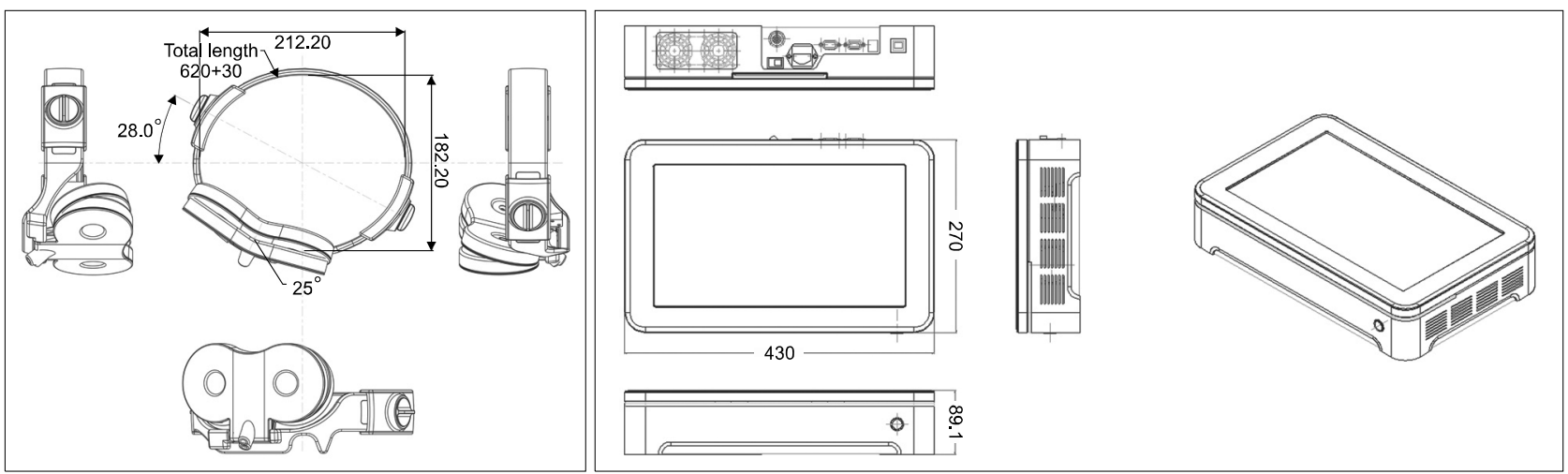

Fig. 1. Drawing of the miniaturized repetitive transcranial magnetic stimulation device (wearable coil and stimulator). 
ing a repeated-measures ANOVA.

\section{RESULTS}

The main demographic and clinical variables of the participants are presented in Table 1. We found no significant differences across the three groups at baseline point. A repeated-measures ANOVA revealed a significant main effect of time on differences between the pre-rTMS and post-rTMS HAM-D scores $(\mathrm{F}(1,44)=94.567, p=0.000$, $\left.\eta_{p}{ }^{2}=0.682\right)$, as well as a significant interaction between rTMS group and time $\left(\mathrm{F}(2,44)=5.447, p=0.008, \eta_{p}{ }^{2}=\right.$ 0.198). ANOVA revealed that changes in HAM-D scores differed significantly among the three groups $(F(2,44)=$ 5.447, $\left.p=0.008, \eta_{p}^{2}=0.198\right)$. Post-hoc analysis showed significant change in HAM-D scores in the miniaturized and standard rTMS compared to sham rTMS (miniaturized vs. sham, $p=0.012$; standard vs. sham, $p=0.049$ ). There were no significant differences in HAM-A or BDI scores among the three groups (Fig. 2). In terms of response and remission rates, eight participants were found to be responders; four were remitters (standard rTMS, four responders and two remitters; miniaturized rTMS, four responders and one remitter; sham rTMS, one remitter). There were no significant differences in responder or remitter ratio among the three groups.

Independent $t$ tests revealed that there were no significant differences in individual MT between the standard and miniaturized rTMS groups. In the miniaturized rTMS group, stimulation was applied at $57.0 \pm 16.4 \%$ of the individual MT. The percentage of MT in all the miniaturized rTMS group was below $90 \%$.

There were no significant correlations between the stimulation intensity and changes of clinical outcomes. No statistically significant differences were found in SDS or VAS scores between the three groups.

\section{DISCUSSION}

To the best of our knowledge, the present study is the first to evaluate the clinical efficacy of miniaturized rTMS in depression. Our findings demonstrate that miniaturized rTMS was comparable to standard rTMS in terms of clinical change and improvement over sham rTMS treatment. The efficacy of miniaturized rTMS treatment was not associated with individual MT levels.

Focusing on efficacy and safety, our results revealed that subthreshold rTMS stimulation is as effective as suprathreshold stimulation in the treatment of depression. A

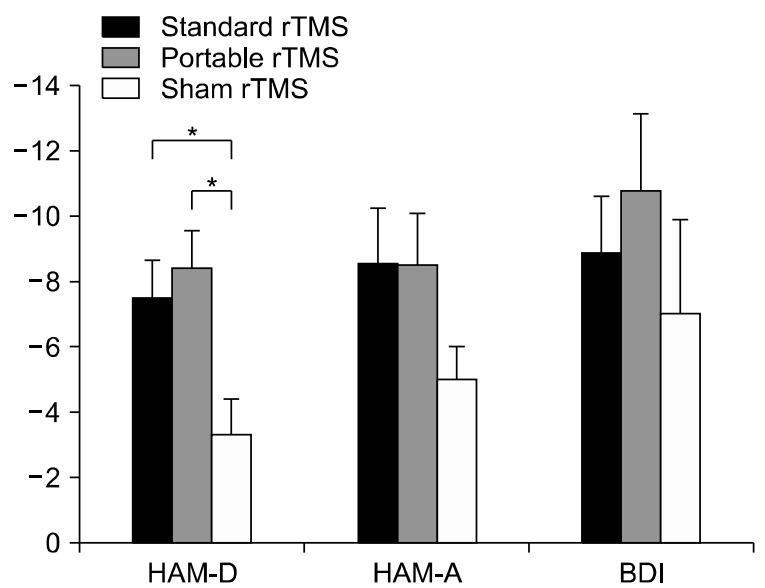

Fig. 2. Changes in the Hamilton Rating Scale for Depression (HAM-D), the Hamilton Rating Scale for Anxiety (HAM-A), and the Beck Depression Inventory (BDI) scores after each repetitive transcranial magnetic stimulation (rTMS) treatment.

Table 1. Demographic variables and clinical measures among the standard, miniaturized and sham rTMS groups at baseline

\begin{tabular}{|c|c|c|c|c|}
\hline Variable & Standard rTMS $(\mathrm{n}=16)$ & Miniaturized rTMS $(n=17)$ & Sham rTMS $(n=14)$ & Significance \\
\hline Sex (male/female) & $3 / 13$ & $4 / 13$ & $4 / 10$ & $\chi^{2}=0.402, p=0.818$ \\
\hline Age (yr) & $34.69 \pm 13.77$ & $33.35 \pm 8.84$ & $36.71 \pm 11.79$ & $\mathrm{~F}(44,2)=0.325, p=0.724$ \\
\hline CGI-S & $4.38 \pm 0.96$ & $4.47 \pm 1.07$ & $4.07 \pm 1.00$ & $\chi^{2}=0.927, p=0.629$ \\
\hline HAM-D & $21.44 \pm 5.21$ & $21.35 \pm 4.23$ & $19.02 \pm 6.40$ & $\mathrm{~F}(44,2)=0.995, p=0.378$ \\
\hline HAM-A & $24.98 \pm 6.39$ & $21.71 \pm 5.39$ & $21.14 \pm 8.34$ & $\mathrm{~F}(44,2)=1.485, p=0.238$ \\
\hline BDI & $28.50 \pm 12.20$ & $31.18 \pm 10.70$ & $27.79 \pm 13.11$ & $\mathrm{~F}(44,2)=0.356, p=0.703$ \\
\hline SDS & $22.50 \pm 4.97$ & $23.41 \pm 5.28$ & $18.93 \pm 9.43$ & $\chi^{2}=1.475, p=0.478$ \\
\hline VAS & $2.88 \pm 2.87$ & $3.12 \pm 3.14$ & $4.29 \pm 3.85$ & $\chi^{2}=1.304, p=0.521$ \\
\hline
\end{tabular}

Values are presented as number only or mean \pm standard deviation.

rTMS, repetitive transcranial magnetic stimulation; CGI-S, Clinical Global Impressions scale for Severity; HAM-D, Hamilton Rating Scale for Depression; HAM-A, Hamilton Rating Scale for Anxiety; BDI, Beck Depression Inventory; SDS, Sheehan Disability Scale; VAS, visual analogue scale. 
significant decrease in HAM-D scores was observed here in participants treated with the miniaturized rTMS compared to sham. There were no differences in clinical change between standard rTMS and miniaturized rTMS treatments. There were no differences in terms of safety among the three groups. These results may seem to contradict previous studies which have suggested that suprathreshold stimulation more effectively modulates excitability and induces clinical improvement than subthreshold stimulation. ${ }^{12,14)}$ However, another study indicated that low intensity rTMS modulates resting state functional connectivity in a rat model. ${ }^{25)}$ In human rTMS, subthreshold rTMS at a high frequency also induced facilitatory effects on corticospinal excitability. ${ }^{15,16)}$ It is therefore possible that changes in resting state are induced by low intensity rTMS, leading to improvements to depressive symptoms.

To prevent excessive coil heating, miniaturized rTMS was administered at subthreshold intensity, irrespective of individual MT in the present study. Novel rTMS techniques using low field magnetic stimulation also have been recently introduced. For example, low field magnetic stimulation synchronized to an individual's alpha frequency was previously found to be effective and safe for treating MDD. ${ }^{21,26)}$ Considering these findings and the results reported here, low intensity magnetic stimulation may have antidepressant effects which depend on the form of rTMS used. Future studies are needed to clarify the effects of subthreshold magnetic stimulation in MDD and to reveal more clinically effective forms of low intensity magnetic stimulation.

rTMS treatment is an onerous and time-demanding clinical method. Patients often find it difficult to complete the recommended course, as outpatient treatment requires daily stimulation for 2 to 3 weeks. Previous guidelines clarified that medically responsible physician and personnel trained to manage a seizure or a syncope is required, and there should be full access to emergency treatment. ${ }^{27)}$ If these conditions are fulfilled and the problem of coil replacement is solved, miniaturized rTMS devices can offer a viable alternative eliminating the need for regular outpatient rTMS clinic visits. The development of miniaturized, noninvasive stimulation devices may thus offer an economical and convenient way to deliver cortical stimulation, improving patients' treatment compliance and enhancing clinical outcomes in patients with MDD.
Despite its strengths, the present study has some limitations worth addressing. First, the present study was a single-blind, not double-blind study, and has a relatively small sample size. Second, suprathreshold and subthreshold stimulation were performed in different devices. The spatial resolution or effect of two devices may be different. Further, we used stimulation intensity as $70 \%$ of maximum stimulator output in miniaturized rTMS group. Although the intensities were subthreshold for all individuals, an individually adapted value such as $90 \%$ of the participant's MT was not used for subthreshold stimulation. It should be considered that our study did not compare suprathreshold and subthreshold stimulation in same devices and constant percentage of MT. Third, to control for the influence of possible environmental factors, all rTMS treatments were conducted in the same outpatient rTMS room. Our study thus cannot answer whether miniaturized rTMS is effective when patients use this device independently and in their own homes. Fourth, having to replace coils frequently may negate the advantage of miniaturized rTMS in home treatment. Device development should be needed to improve patient's convenience. Fifth, sham stimulation with only acoustic sensation may not convince participants that they received treatment. Future studies should provide blinding questionnaire at the end of treatment.

Our findings presented here suggest that miniaturized rTMS, utilizing subthreshold stimulation, has significant antidepressant effects in patient with depression which are comparable to standard rTMS treatment. Future studies should investigate the efficacy of self-treatment using miniaturized rTMS at home to further determine the clinical applicability of this new treatment modality.

\section{- Acknowledgments}

This research was supported by a grant of the Korea Health Technology R\&D Project through the Korea Health Industry Development Institute (KHIDI), funded by the Ministry of Health \& Welfare, Republic of Korea (grant number: HI17C2272). REMED (Daejeon, Korea) provided partial funding for the present study.

\section{- Conflicts of Interest}

No potential conflict of interest relevant to this article was reported.

Sejin Yoon works as a principle engineer at REMED 
Co., Ltd. That had no influence in study design, data collection and analysis, decision to publish, or preparation of the manuscript.

\section{REFERENCES}

1. Olfson M, Marcus SC, Druss B, Elinson L, Tanielian T, Pincus $\mathrm{HA}$. National trends in the outpatient treatment of depression. JAMA 2002;287:203-209.

2. George MS, Aston-Jones G. Noninvasive techniques for probing neurocircuitry and treating illness: vagus nerve stimulation (VNS), transcranial magnetic stimulation (TMS) and transcranial direct current stimulation (tDCS). Neuropsychopharmacology 2010;35:301-316.

3. Pascual-Leone A, Rubio B, Pallardó F, Catalá MD. Rapid-rate transcranial magnetic stimulation of left dorsolateral prefrontal cortex in drug-resistant depression. Lancet 1996;348. 233-237.

4. Padberg F, George MS. Repetitive transcranial magnetic stimulation of the prefrontal cortex in depression. Exp Neurol 2009;219:2-13.

5. George MS, Wassermann EM, Williams WA, Callahan A, Ketter TA, Basser $\mathrm{P}$, et al. Daily repetitive transcranial magnetic stimulation (rTMS) improves mood in depression. Neuroreport 1995;6:1853-1856.

6. O'Reardon JP, Solvason HB, Janicak PG, Sampson S, Isenberg $\mathrm{KE}, \mathrm{Nahas} \mathrm{Z}$, et al. Efficacy and safety of transcranial magnetic stimulation in the acute treatment of major depression: a multisite randomized controlled trial. Biol Psychiatry 2007;62: 1208-1216.

7. Kumar S, Singh S, Kumar N, Verma R. The effects of repetitive transcranial magnetic stimulation at dorsolateral prefrontal cortex in the treatment of migraine comorbid with depression: a retrospective open study. Clin Psychopharmacol Neurosci 2018;16:62-66.

8. Wang J. Mental health treatment dropout and its correlates in a general population sample. Med Care 2007;45:224-229.

9. Melartin TK, Rytsälä HJ, Leskelä US, Lestelä-Mielonen PS, Sokero TP, Isometsä ET. Continuity is the main challenge in treating major depressive disorder in psychiatric care. J Clin Psychiatry 2005;66:220-227.

10. George MS, Lisanby SH, Avery D, McDonald WM, Durkalski V, Pavlicova M, et al. Daily left prefrontal transcranial magnetic stimulation therapy for major depressive disorder: a sham-controlled randomized trial. Arch Gen Psychiatry 2010; 67:507-516.

11. Berlim MT, van den Eynde F, Tovar-Perdomo S, Daskalakis ZJ. Response, remission and drop-out rates following high-frequency repetitive transcranial magnetic stimulation (rTMS) for treating major depression: a systematic review and meta-analysis of randomized, double-blind and sham-controlled trials. Psychol Med 2014;44:225-239.

12. Padberg F, Zwanzger $P$, Keck ME, Kathmann N, Mikhaiel $P$,
Ella $\mathrm{R}$, et al. Repetitive transcranial magnetic stimulation (rTMS) in major depression: relation between efficacy and stimulation intensity. Neuropsychopharmacology 2002;27: 638-645.

13. Boutros NN, Gueorguieva R, Hoffman RE, Oren DA, Feingold A, Berman RM. Lack of a therapeutic effect of a 2-week subthreshold transcranial magnetic stimulation course for treatment-resistant depression. Psychiatry Res 2002;113:245-254.

14. Lang N, Harms J, Weyh T, Lemon RN, Paulus W, Rothwell JC, et al. Stimulus intensity and coil characteristics influence the efficacy of rTMS to suppress cortical excitability. Clin Neurophysiol 2006;117:2292-2301.

15. Sommer M, Rummel M, Norden C, Rothkegel H, Lang N, Paulus W. Mechanisms of human motor cortex facilitation induced by subthreshold 5- $\mathrm{Hz}$ repetitive transcranial magnetic stimulation. J Neurophysiol 2013;109:3060-3066.

16. Rothkegel $\mathrm{H}$, Sommer M, Paulus W. Breaks during $5 \mathrm{~Hz}$ rTMS are essential for facilitatory after effects. Clin Neurophysiol 2010;121:426-430.

17. Yoo SW, Kim YS, Noh JS, Oh KS, Kim CH, NamKoong K, et al. Validity of Korean version of the MINI-International Neuropsychiatric Interview. Anxiety Mood 2006;2:50-55.

18. Hamilton M. A rating scale for depression. J Neurol Neurosurg Psychiatry 1960;23:56-62.

19. Hamilton M. The assessment of anxiety states by rating. Br Med Psychol 1959;32:50-55.

20. Beck AT, Ward CH, Mendelson M, Mock J, Erbaugh J. An inventory for measuring depression. Arch Gen Psychiatry 1961; 4:561-571.

21. Leuchter AF, Cook IA, Feifel D, Goethe JW, Husain M, Carpenter LL, et al. Efficacy and safety of low-field synchronized transcranial magnetic stimulation (sTMS) for treatment of major depression. Brain Stimul 2015;8:787-794.

22. Sheehan DV, Harnett-Sheehan K, Raj BA. The measurement of disability. Int Clin Psychopharmacol 1996;11 Supp/ 3:89-95.

23. Ahearn EP. The use of visual analog scales in mood disorders: a critical review. J Psychiatr Res 1997;31:569-579.

24. Herwig U, Padberg F, Unger J, Spitzer M, Schönfeldt-Lecuona C. Transcranial magnetic stimulation in therapy studies: examination of the reliability of "standard" coil positioning by neuronavigation. Biol Psychiatry 2001;50:58-61.

25. Seewoo BJ, Feindel KW, Etherington SJ, Rodger J. Resting-state fMRI study of brain activation using low-intensity repetitive transcranial magnetic stimulation in rats. Sci Rep 2018;8:6706.

26. Jin Y, Phillips B. A pilot study of the use of EEG-based synchronized transcranial magnetic stimulation (sTMS) for treatment of major depression. BMC Psychiatry 2014;14:13.

27. Rossi S, Hallett M, Rossini PM, Pascual-Leone A; Safety of TMS Consensus Group. Safety, ethical considerations, and application guidelines for the use of transcranial magnetic stimulation in clinical practice and research. Clin Neurophysiol 2009;120:2008-2039. 